01,13

\title{
Распыление атомов металлов кильватерным потенциалом, возбужденным пучком электронов
}

\author{
(C) Н.П. Калашников \\ Национальный исследовательский ядерный университет „МИФИ“, \\ Москва, Россия \\ E-mail: kalash@mephi.ru
}

Поступила в Редакцию 22 января 2022 г.

В окончательной редакции 29 января 2022 г.

Принята к публикации 29 января 2022 г.

\begin{abstract}
Рассматривается процесс распыления атомов металла при коронном разряде. При движении электрона в среде с некоторой скоростью, экранирование заряда происходит с запаздыванием в пространстве и во времени, что приводит к возникновению кильватерного потенциала. Возбужденные колебания кильватерного заряда приводят к появлению дополнительных сил. Потери энергии движущейся частицей на единице пути определяются работой, производимой силой торможения, которая действует на частицу со стороны создаваемого ею в среде кильватерного потенциала. В работе рассматривается воздействие кильватерного потенциала на ионы (атомы) матрицы решетки. Используется известное выражение для кильватерного потенциала, возбуждаемого заряженной частицей, движущейся с энергией, большей энергии Ферми. Получено выражение для сечения распыления атомов металла под действием кильватерного потенциала, возбужденного электронным пучком. Показано, что результат распыления не зависит от знака заряда падающей частицы (электрона или иона).
\end{abstract}

Ключевые слова: коронный разряд, наночастицы, металлическая поверхность, неупругое рассеяние, кильватерный потенциал.

DOI: $10.21883 /$ FTT.2022.05.52328.282

\section{1. Введение}

Процессы, связанные с электрическими разрядами [1], находят свое применение в современных технологиях, поскольку благодаря им можно создавать наноразмерные элементы. В то же время, поскольку возникает необходимость создавать элементы еще меньшего размера, приходится применять и коронный разряд, поскольку в этом случае можно получить элементы в единицы и доли нанометров [2,3]. При протекании коронного разряда атомы и молекулы вещества, из которого изготовлены электроды, оказываются в газовой среде. На существование такого испарения указывает появление на месте соприкосновения шнура плазмы с поверхностью электрода соответствующего углубления [4].

В настоящей статье предложена теоретическая модель распыления металла. Показано, что причиной аномальной эмиссии атомов может являться взаимодействие атомов (ионов) металла с кильватерным потенциалом [5], возбуждаемым падающими электронами или ионами коронного разряда.

\section{2. Кильватерный потенциал, возбужденный движущимися в веществе электронами}

Пролетающая через вещество быстрая заряженная частица поляризует молекулы вещества, создавая в каждой из них переменный дипольный момент. Так возникают поляризационные токи внутри вещества. Возбуждение вещества быстрой заряженной частицей происходит за счет кинетической энергии падающей частицы. Если частоты возбуждений порядка или меньше оптических частот, то такие возбуждения длинноволновые и могут рассматриваться в макроскопической электродинамике [6]. Основную роль при этом играют медленно затухающие со временем, т.е. долгоживущие возбуждения.

В однородной и изотропной среде возможно существование поперечных электромагнитных волн, волновой вектор $\mathbf{k}$ которых удовлетворяет дисперсионному уравнению

$$
k^{2}-\left(\frac{\omega}{c}\right)^{2} \varepsilon^{t r}(\mathbf{k}, \omega)=0
$$

и продольных электромагнитных волн, волновой вектор которых определяется уравнением

$$
\varepsilon^{l}(\mathbf{k}, \omega)=0
$$

Обращение в нуль диэлектрической проницаемости является условием существования продольных электромагнитных волн, т. е. колебания кильватерного заряда [5] возникают в результате возбуждения продольных электромагнитных волн движущимся зарядом.

В случае электрона, движущегося с некоторой скоростью в среде, экранирование заряда происходит с запаздыванием в пространстве и во времени, что приводит к возникновению кильватерного потенциала [5]. 
Колебания кильватерной плотности заряда создают соответствующий этой плотности заряда кильватерный потенциал. Уравнение для Фурье-образа кильватерного потенциала имеет вид

$$
\Delta \varphi_{\omega}(\mathbf{r}, \omega)=-4 \pi \rho_{\omega}(\mathbf{r}, \omega)
$$

так как частоты колебаний соответствуют нулям $\varepsilon^{l}(\mathbf{k}, \omega)$.

Созданное колебаниями кильватерного заряда поле действует на внешнюю частицу и на ионы вещества. Рассмотрим колебания кильватерного заряда, соответствующие паре нулей $\varepsilon(\omega)$, чтобы определить связанную с этим механизмом силу торможения. Для широкого класса твердых тел возможно существование коллективных колебаний электронов вещества - плазмонов, частота которых соответствует обращению в нуль диэлектрической проницаемости

$$
\varepsilon(\omega)=1-\frac{\omega_{p}^{2}}{\omega\left(\omega+i \gamma_{p}\right)}, \quad \omega_{p}^{2}=\frac{4 \pi n_{e} e^{2}}{m} .
$$

(Для металлов величина $n_{e}$ соответствует числу электронов проводимости в единице объема.)

Величина $\rho(\mathbf{r}, t)$ представляет собой дополнительную переменную плотность заряда, образующуюся в веществе в результате заданного движения в веществе зарядов

$$
\begin{aligned}
\rho(\mathbf{r}, t)= & \frac{z e \omega_{p}}{v} \sin \left\{\omega_{p}\left(t-\frac{z}{v}\right)\right\} \\
& \times \exp \left\{-\gamma_{p}\left(t-\frac{z}{v}\right)\right\} \delta(\boldsymbol{\rho}) \theta(v t-z) .
\end{aligned}
$$

Таким образом, возбужденные полем внешних, не входящих в состав вещества частиц (падающего электрона или иона), колебания кильватерного заряда приводят к появлению дополнительных сил. В частности, на падающую свободную частицу с зарядом $Z_{1}$ е в точке $\mathbf{v}$ в момент времени $t$ со стороны кильватерного заряда, созданного той же самой частицей, действует сила $\mathbf{F}(t)=-Z_{1} e \operatorname{grad} \varphi(\mathbf{v} t, t)$.

Потери энергии движущейся частицей на единице пути определяются работой, производимой силой торможения, которая действует на частицу со стороны создаваемого ею в среде электромагнитного поля (кильватерного потенциала) [6,7]:

$$
\frac{d E}{d z}=\frac{\mathbf{v F}}{v}=\frac{Z_{1} e}{v}(\mathbf{v E})=-\frac{Z_{1} e^{2} 4 \pi n_{e}}{m v^{2}} \ln \left(\frac{v q_{\max }}{\omega_{p}}\right) .
$$

Итак, в случае электрона (иона), движущегося с некоторой скоростью в среде, экранирование заряда происходит с запаздыванием в пространстве и во времени, что приводит к возникновению „кильватерного потенциала“. Для электрона (иона), движущегося в металле, экранирование и кильватерный эффект [7] хорошо описываются возбуждением виртуальных плазмонов [5]. Нойфелд и
Ричи [8] получили выражение для кильватерного потенциала для заряженной частицы, движущейся с энергией, большей энергии Ферми:

$$
\varphi=-\frac{2 Z_{1} e}{v} \omega_{p} \sin \left(\frac{\omega_{p}}{v} z\right) K_{0}\left(\rho \frac{\omega_{p}}{v}\right),
$$

где $K_{0}\left(\rho \frac{\omega_{p}}{v}\right)$ - модифицированная функция Бесселя второго рода, $z$ и $\rho$ - продольная и поперечная координаты. Потенциальную энергию взаимодействия иона решетки с зарядом $Z_{2} e$ можно записать в виде

$$
U(\rho, z)=Z_{2} e \varphi=-\frac{2 Z_{1} Z_{2} e^{2}}{v} \omega_{p} \sin \left(\frac{\omega_{p}}{v} z\right) K_{0}\left(\rho \frac{\omega_{p}}{v}\right)
$$

Вагер и Джеммел [9] предложили следующую формулу для кильватерного потенциала:

$$
\begin{aligned}
\varphi(\rho, z & -\omega t)=-\frac{Z_{1} e \omega_{p}}{v} \int_{0}^{\infty} \sin \left(\frac{\omega_{p}}{v} \xi\right) \\
& \times\left[\rho^{2}+\left(\frac{\hbar}{m v}\right)^{2}+(\xi+z-v t)^{2}\right]^{-1 / 2} d \xi .
\end{aligned}
$$

В работе [10] была предложена формула для кильватерного потенциала, записанная через диэлектрическую проницаемость

$$
\begin{aligned}
\varphi= & -\frac{Z_{1} e}{\pi v} \int_{0}^{\infty} \xi d \xi J_{0}(\xi \rho) \\
& \times \int_{-\infty}^{\infty} d \omega \exp \left(\frac{i \omega(z-v t)}{v}\right) \frac{1}{k^{2}} \frac{1}{\varepsilon(k, \omega)},
\end{aligned}
$$

где $\rho=\left(x^{2}+y^{2}\right)^{1 / 2}$ и $k^{2}=\xi^{2}+\frac{\omega^{2}}{v^{2}}$.

Из выражения (10) может быть получена формула Нойфелда и Ричи (7). Если $\frac{1}{\varepsilon(k, \omega)}=i \pi \delta(\varepsilon(k, \omega))$ [6] и диэлектрическую проницаемость имеет вид (4), то интегрирование по $d \omega$ дает выражение

$$
\begin{aligned}
\varphi & =-\frac{Z_{1} e}{2 v} i \int_{0}^{\infty} \xi d \xi J_{0}(\xi \rho) \omega_{p} \exp \left(\frac{i \omega_{p}(z-v t)}{v}\right) \frac{1}{\xi^{2}+\omega_{p}^{2} / v^{2}} \\
& =-\frac{Z_{1} e}{2 v} \omega_{p} K_{0}\left(\frac{\omega_{p}}{v} \rho\right) \sin \left(\frac{\omega_{p}(z-v t)}{v}\right) \theta(v t-z),
\end{aligned}
$$

которое совпадает с результатом Нойфелда и Ричи (7).

\section{3. Распыление ионов металлов кильватерным потенциалом}

Рассмотрим теперь воздействие кильватерного потенциала на ионы (атомы) матрицы решетки. Для электрона (иона), движущегося в металле, экранирование 
и кильватерный эффект [7] хорошо описываются выражением (11). Для иона (атома) вещества мишени вероятность перехода в единицу времени (в единице объема) в первом приближении теории возмущений [11]:

$$
d P_{f i}=\frac{2 \pi}{\hbar}\left|\left\langle\Phi_{f}^{*}|W| \Phi_{i}\right\rangle\right|^{2} \frac{m k_{f} d \Omega}{(2 \pi \hbar)^{2}},
$$

где матричный элемент определяется интегралом

$$
\left\langle\Phi_{f}^{*}|W| \Phi_{i}\right\rangle=\int \varphi_{f}^{*}(\xi) W(\mathbf{r}, \xi) \varphi_{i}(\xi) d \xi \exp \left(i\left(\mathbf{k}_{i}-\mathbf{k}_{f}\right) \mathbf{r}\right) d^{3} \mathbf{r}
$$

где $W(\mathbf{r}, \xi)=-\operatorname{Im} \frac{Z_{1} e}{2 v} \omega_{p} K_{0}\left(\frac{\omega_{p}}{v} \rho\right) \exp \left(\frac{\omega_{p}(z-v \xi)}{v}\right)-$ оператор взаимодействия $(8), \varphi_{f}(\xi)$ и $\varphi_{i}(\xi)-$ волновые функции конечного и начального состояний иона в решетке. Интеграл по $d \xi$ имеет вид

$$
\begin{array}{r}
\int_{0}^{\infty} \exp \left(i \varepsilon_{b} \xi+i \frac{p_{i}^{2}}{2 m} \xi\right) \exp \left(-i \omega_{b} \xi-i \frac{p_{f}^{2}}{2 m} \xi\right) \\
=1 / 2 \delta\left(\omega_{p}+\frac{p_{f}^{2}}{2 m}-\varepsilon_{b}+\frac{p_{i}^{2}}{2 m}\right),
\end{array}
$$

где $\varepsilon_{b}$ - энергия связи иона в решетке материала.

Эффективное сечение (упругого и неупругого) рассеяния в первом борновском приближении может быть записано в следующем виде:

$$
d \sigma_{f i}^{(B o h r)}=\left(\frac{m}{2 \pi \hbar^{2}}\right)^{2} \frac{p_{f}}{p_{i}}\left|\left\langle\Phi_{f}^{*}|W| \Phi_{i}\right\rangle\right|^{2} d \Omega
$$

где $p_{f}=\sqrt{p_{i}^{2}+2 m\left(\omega_{p}-\varepsilon_{b}\right)}$.

При вычислении полного сечения воспользуемся квазиклассическим выражением для оптической теоремы [12]:

$$
\begin{aligned}
\sigma_{t o t} & =4 \pi \int_{0}^{\infty} \rho d \rho\left\{1-\cos \left[\frac{1}{\hbar v} \int_{-\infty}^{\infty} U\left(\sqrt{\rho^{2}+z^{2}}\right) d z\right]\right\} \\
& \approx 2 \pi \int_{0}^{\infty} \rho d \rho \frac{1}{(\hbar v)^{2}}\left[\int_{-\infty}^{\infty} U\left(\sqrt{\rho^{2}+z^{2}}\right) d z\right]^{2}
\end{aligned}
$$

Используя для потенциала взаимодействия выражение (8)

$$
U(\rho, z)=Z_{2} e \varphi=-\frac{2 Z_{1} Z_{2} e^{2}}{v} \omega_{p} \sin \left(\frac{\omega_{p}}{v} z\right) K_{0}\left(\rho \frac{\omega_{p}}{v}\right),
$$

после введения безразмерных переменных интегрирования получаем

$$
\sigma_{\text {tot }} \approx \frac{\pi}{2} \frac{1}{\left(\hbar \omega_{p}\right)^{2}} Z_{1}^{2} Z_{2}^{2} e^{4} \int_{0}^{\infty} x d x\left(\int_{0}^{\infty} K_{0}\left(\sqrt{x^{2}+\xi^{2}}\right) \sin \xi d \xi\right)^{2} .
$$

Таким образом, с точностью до численного множителя полное сечение распыления пропорционально

$$
\sigma_{t o t} \sim \frac{1}{\left(\hbar \omega_{p}\right)^{2}} Z_{1}^{2} Z_{2}^{2} e^{4} \theta\left(\hbar \omega_{p}-\varepsilon_{b}\right) .
$$

Из полученного выражения следует, что результат распыления не зависит от знака заряда падающей частицы (электрона или иона).

\section{Благодарности}

Работа выполнена в рамках Программы „Приоритет $-2030^{\varsigma}$.

\section{Конфликт интересов}

Автор заявляет об отсутствии конфликта интересов.

\section{Список литературы}

[1] D. Megyeria, A. Kohuta, Z. Geretovszky. J. Aerosol Sci. 154, 105758 (2021).

[2] J. Niedbalski. Rev. Sci. Instruments 74, 7, 3520 (2003).

[3] Ming-Wei Li, Zheng $\mathrm{Hu}, \mathrm{Xi}$-Zhang Wang, Qiang Wu, Yi Chen. J. Mater. Sci. 39, 1, 283 (2004).

[4] В.А. Курнаев, Ю.С. Протасов, И.В. Цветков. Введение в пучковую электронику. МИФИ, М. (2008). 452 с.

[5] Е.-Х. Оцуки. Взаимодействие заряженных частиц с твердыми телами. Мир, М. (1985). 280 с.

[6] М.И. Рязанов. Введение в электродинамику конденсированного вещества. Физматлит, М. (2002). 320 с.

[7] Л.Д. Ландау, Е.М. Лифшиц. Электродинамика сплошных сред. Наука, М. (1992). T. VIII. 664 с.

[8] J. Neufeld, R.H. Ritchie. Phys. Rev. 98, 1632 (1979).

[9] Z. Vager, D.S. Gemmel. Phys. Rev. Lett. 37, 1352 (1976).

[10] P.M. Echenique, R. Ritchie, W. Brandt. Phys. Rev. B 14, 4808 (1976).

[11] Л.Д. Ландау, Е.М. Лифшиц. Квантовая механика. Нерелятивистская теория. Наука, М. (1989). Т. III. 768 с.

[12] N. Kalashnikov. Coherent Interactions of Charged Particles in Single Crystals. Scattering and Radiative Processes in Single Crystals. Harwood Academic Publishers, London-Paris-N. Y. (1988). 328 p.

Редактор Ю.Э. Китаев 